\title{
TAMANHO DE PARCELA E NÚMERO DE REPETIÇÕES PARA MAMONEIRA EM DIFERENTES ESPAÇAMENTOS ENTRE PLANTAS ${ }^{1}$
}

\author{
ANDRÉ LUIZ PALUDO ${ }^{2}$, SIDINEI JOSÉ LOPES ${ }^{3 *}$, BETANIA BRUM ${ }^{4}$, LINDOLFO STORCK ${ }^{5}$, \\ DANIEL DOS SANTOS $^{6}$, FERNANDO MACHADO HAESBAERT ${ }^{6}$
}

\begin{abstract}
RESUMO - O planejamento adequado de experimentos na cultura de mamoneira é uma das formas de se maximizar as informações da pesquisa. Os objetivos deste trabalho foram determinar o tamanho de parcela e o número de repetições para a cultura de mamoneira e verificar a influência do espaçamento entre plantas no plano experimental. O experimento com o híbrido Sara foi conduzido na Universidade Federal de Santa Maria, em 2010, com espaçamento entre linhas de 1,2 m e espaçamentos entre plantas de 0,4, 0,6 e 0,8 m. Foram avaliados em cada planta o número de rácemos, peso de frutos, comprimento médio dos rácemos e número total de frutos, com identificação da planta pelo número de ordem da fileira e o número da planta dentro da fileira. O tamanho ótimo de parcela é de oito plantas para todos os espaçamentos e para todas as variáveis. Porém, a área do tamanho ótimo de parcela em cada espaçamento depende da área ocupada pela unidade básica. Assim, a área da parcela é de 3,84, 5,76 e 7,68 $\mathrm{m}^{2}$ para os espaçamentos entre plantas de 0,4, 0,6 e 0,8 m, respectivamente. Doze repetições, no delineamento blocos ao acaso, foram suficientes para identificar diferenças entre médias de tratamentos de $27 \%$, com 5\% de probabilidade de erro.
\end{abstract}

Palavras-chave: Ricinus communis L. Precisão experimental. Tamanho de parcela.

\section{PLOT SIZE AND NUMBER OF REPLICATIONS FOR CASTOR BEAN AT DIFFERENT SPACING BETWEEN PLANTS}

\begin{abstract}
Proper planning of experiments in the culture of castor bean is one of the ways to maximize the research information. The aim of this study was to determine the optimum plot size and number of replications and the influence of spacing between plants in experiments involving castor bean crops. The experiment with the hybrid Sara was conducted at the Federal University of Santa Maria in 2010 with a spacing of $1.2 \mathrm{~m}$ between rows and between plants of $0.4,0.6$ and $0.8 \mathrm{~m}$. Each plant was evaluated taking into account: the number of racemes, fruit weight, the average length of the racemes, and fruit number, with the plant identification by the order number of the row and the number of plants within the row. The optimum plot size is eight plants for all spacings and all variables. However, the area of optimum plot size inside the spacing depends on the area occupied by the basic unit. Thus, the plot area is $3.84,5.76$ and $7.68 \mathrm{~m}^{2}$ for each spacing of $0.4,0.6$ and $0.8 \mathrm{~m}$, respectively. Twelve replications in randomized block design, are sufficient to identify, as significant at $5 \%$ probability, differences between treatment means of $27 \%$.
\end{abstract}

Keywords: Ricinus communis L. Experimental accuracy. Plot size.

\footnotetext{
*Autor para correspondência

${ }^{1}$ Recebido para publicação em 20/05/2014; aceito em 31/08/2015.

Trabalho de Dissertação de Mestrado em Agronomia do primeiro autor.

${ }^{2}$ Engenheiro Agrônomo, M.Sc. Universidade Federal de Santa Maria, Santa Maria (RS), Brasil; agropaludo@gmail.com.

${ }^{3}$ Professor Associado da Universidade Federal de Santa Maria, CCR, Depto. Fitotecnia, A. Roraima, nº 1000, 97105-900 Santa Maria (RS), Brasil; sjlopes@pq.cnpq.br.

${ }^{4}$ Professora Adjunta da Universidade Tecnológica Federal do Paraná (UFTPR), Pato Branco (PR), Brasil; bbufsm@gmail.com.

${ }^{5}$ Professor Visitante da Universidade Tecnológica Federal do Paraná (UFTPR), Pato Branco (PR), Brasil; lindolfostorck@gmail.com.

${ }^{6}$ Doutorando do Programa de Pós-Graduação em Agronomia da Universidade Federal de Santa Maria, Santa Maria (RS), Brasil; danielsantosagro@gmail.com, fhaesbaert@gmail.com.
} 


\section{INTRODUÇÃO}

A ricinicultura tem como principal importância econômica a produção de óleo, apresentando inúmeras aplicações nas indústrias química e farmacêutica. É uma cultura promissora para a produção de biodiesel, pois o óleo de mamona é o único $100 \%$ solúvel em álcool (COMISSÃO ESPECIAL DE BIO-ENERGIA, 2006).

A área plantada no Brasil em 2013/14 foi de 101,3 mil hectares, com produtividade média de 441 $\mathrm{kg} \mathrm{ha}^{-1}$, cuja produção não ultrapassou 45 mil toneladas (CONAB, 2014), a menor dos últimos 30 anos. A baixa produtividade de grãos dos produtores brasileiros se deve a vários fatores, dentre eles a falta de melhorias no sistema de cultivo. O espaçamento entre fileiras e a população de plantas afetam a produtividade de mamoneira (CARVALHO et al., 2010). Otimizar essas técnicas culturais pode levar a maximização econômica da produção com simplicidade de aplicação (PETINARI et al., 2012).

O cultivo intensivo ou em novas áreas necessita de estudos de técnicas experimentais adequadas para a condução de pesquisas visando a melhoria da produtividade, tais como o tamanho de parcela e o número de repetições. Além disso, o plano experimental depende da cultura e das características avaliadas dos tratamentos e das condições ambientais de cada experimento (GOMEZ; GOMEZ, 1984; STORCK et al., 2011).

Durante a fase de planejamento, o pesquisador deve considerar os diversos fatores que induzem ao erro experimental, dentre eles a heterogeneidade do material experimental, o qual compõe os tratamentos. $\mathrm{O}$ erro experimental gerado pela heterogeneidade das unidades experimentais pode ser quantificado através da execução do experimento em branco ou ensaio de uniformidade para adequar a área experimental ao tamanho e ao número de repetições e de tratamentos com a precisão desejada, maximizando a qualidade das informações obtidas na área experimental disponível (HOSHMAND, 2006; STORCK et al., 2011).

Para a estimativa do tamanho ótimo de parcela deve-se conhecer o índice de heterogeneidade da característica medida. Este índice varia em função das diferenças de fertilidade do solo, drenagem, relevo, manejo ou resíduos de culturas anteriores e aplicação de adubos para a implantação do experimento (STEEL et al., 1997; STORCK et al., 2011). Hatheway (1961) sugeriu uma fórmula para o cálculo do tamanho de parcela que depende do índice de heterogeneidade, cuja obtenção é um tanto complexa, mas de uso comum tanto para ensaios em branco quanto para experimentos que envolvam vários tratamentos (ALVES; SERAPHIN, 2004)

Visando a melhoria da precisão experimental, na cultura de mamona, Cargnelutti Filho et al. (2010) observaram que há aumento do tamanho da amostra para estimar caracteres de semente, plântula, planta adulta e produção, nessa ordem, o que mostra a necessidade de maior tamanho da amostra para os caracteres que permanecem por mais tempo sob atuação do ambiente.

$\mathrm{O}$ uso adequado do espaçamento entre plantas de mamoneira é fundamental para otimizar a produtividade da cultura e o adequado planejamento dos experimentos para qualificar os resultados de pesquisas. Os objetivos deste estudo foram determinar o tamanho adequado de parcela e o número de repetições em diferentes espaçamentos entre plantas na cultura de mamona.

\section{MATERIAL E MÉTODOS}

Os dados de produção da cultura de mamona, híbrido comercial Sara, foram coletados em experimento no Departamento de Fitotecnia da Universidade Federal de Santa Maria, com coordenadas 29 43' 23 ''S e $53^{\circ} 43^{\prime} 15^{\prime}$ 'W e altitude de $95 \mathrm{~m}$.

A semeadura da cultura foi realizada colocando duas sementes por cova, no dia 19 de outubro de 2010. Vinte dias após a semeadura (DAS) foi realizado o desbaste manual, deixando uma planta por cova. No momento da semeadura fora realizado a adubação de base, seguindo as recomendações de adubação e calagem para a cultura de mamona no Rio Grande do Sul (SCIVITTARO; PILLON, 2006). Foram usadas três áreas próximas, cada uma com 12 fileiras de 14,4 m espaçadas de 1,2 m, variando o espaçamento entre plantas na fileira em $0,40 \mathrm{~m}$ (20800 plantas ha $\left.{ }^{-1}\right), 0,60 \mathrm{~m}\left(13900\right.$ plantas ha $\left.{ }^{-1}\right) \mathrm{e}$ $0,80 \mathrm{~m}$ (10400 plantas ha $\left.^{-1}\right)$.

Foram realizadas duas colheitas de acordo com a maturação dos rácemos, no qual após o corte foram acondicionados em sacos de papel devidamente identificados. A secagem dos rácemos foi realizada em estufa a temperatura de $40^{\circ} \mathrm{C}$ por 24 horas para posterior pesagem. Foram avaliados em cada planta o número de rácemos, o peso de frutos $\left(\mathrm{g}\right.$ planta $\left.{ }^{-1}\right)$, o comprimento médio dos rácemos $(\mathrm{cm})$ e o número total de frutos.

Devido a variação do espaçamento entre plantas nas três áreas obteve-se: 432 plantas (12 fileiras com 36 plantas) no espaçamento $0,4 \mathrm{~m} ; 288$ plantas (12 fileiras com 24 plantas) no espaçamento $0,6 \mathrm{~m}$; e 216 plantas (12 fileiras com 18 plantas) no espaçamento $0,8 \mathrm{~m}$. A planta foi considerada como sendo a unidade básica em cada uma das três áreas. Foram planejados diferentes tamanhos de parcela pelo agrupamento de X1 fileiras de plantas adjacentes (largura) e X2 plantas adjacentes na fila (comprimento), de modo que $\mathrm{X} 1 * \mathrm{X} 2=\mathrm{X}$ é o tamanho de parcela em número de plantas. Os tamanhos $\left(\mathrm{X}_{1} * \mathrm{X}_{2}\right)$ planejados foram $1 * 1,1 * 2,1 * 3,1 * 4,1 * 6$, $1 * 9,2 * 2,2 * 3,2 * 4,2 * 6,2 * 9,3 * 3,3 * 4,3 * 6$ e $3 * 9$

Foram calculados o número de parcelas $(\mathrm{N})$, com $\mathrm{X}$ unidades básicas (UB) de tamanho $(\mathrm{N}=432$ / $\mathrm{X}$, no espaçamento $0,4 \mathrm{~m} ; \mathrm{N}=288 / \mathrm{X}$ no espaçamento 
0,6 m; e, $\mathrm{N}=216 / \mathrm{X}$ no espaçamento $0,8 \mathrm{~m}$ ), média das parcelas $[\mathrm{M}(\mathrm{x})]$, com X UB de tamanho, variância entre parcelas $[\mathrm{V}(\mathrm{x})]$ de $\mathrm{X}$ UB de tamanho, variância por $\mathrm{UB}\left[\mathrm{VU}(\mathrm{x})=\mathrm{V}(\mathrm{x}) / \mathrm{X}^{2}\right]$, calculada entre as parcelas de $\mathrm{X} U \mathrm{UB}$, e coeficiente de variação entre as parcelas $[\mathrm{CV}(\mathrm{x})]$ de X UB de tamanho.

Foi estimado, para cada área e para cada característica avaliada, o índice de heterogeneidade (b), segundo a relação empírica de Smith (1938), qual seja: $\mathrm{VU}(\mathrm{x})=\mathrm{V}_{1} / \mathrm{X}^{\mathrm{b}}$. O valor de $\mathrm{b}$ foi estimado como um coeficiente de regressão linear pela transformação logarítmica da função e a estimação ponderada pelos graus de liberdade associados aos 15 tipos de parcelas planejadas (STEEL et al., 1997). No modelo, $\mathrm{V}_{1}$ é o parâmetro para estimar a variância entre as parcelas de uma UB. De forma semelhante, também foram estimados os parâmetros da função $\mathrm{CV}(\mathrm{x})=$ $\mathrm{A} / \mathrm{X}^{\mathrm{B}}$.

Utilizando-se as estimativas dos parâmetros das funções $\mathrm{VU}(\mathrm{x})=\mathrm{V}_{1} / \mathrm{X}^{\mathrm{b}}$ e $\mathrm{CV}(\mathrm{x})=\mathrm{A} / \mathrm{X}^{\mathrm{B}}$, e a média das parcelas com uma UB $\left(\mathrm{M}_{1}\right)$, foram obtidas as estimativas do tamanho ótimo de parcela (Xo) pelos métodos de máxima curvatura modificada (MO) e máxima curvatura em função da VU (MV), respectivamente:

$$
\mathrm{MO}=\exp \left\{[ 1 / ( 2 \mathrm { B } + 2 ) ] \operatorname { l n } \left[\mathrm{A}^{2} \mathrm{~B}^{2}(2 \mathrm{~B}+1) /(\mathrm{B}+\right.\right.
$$

2)]\} (MEIER; LESSMAN, 1971);

$\mathrm{MV}=\exp \left\{[1 /(2 \mathrm{~b}+2)] \ln \left[\mathrm{bV} 1^{2}(2 \mathrm{~b}+1) /(\mathrm{b}+\right.\right.$

2)]\} (THOMAS, 1974).

Com as mesmas estimativas dos parâmetros das funções, $\mathrm{VU}(\mathrm{x})=\mathrm{V}_{1} / \mathrm{X}^{\mathrm{b}}$ e $\mathrm{CV}(\mathrm{x})=\mathrm{A} / \mathrm{X}^{\mathrm{B}}$ também foi obtida a estimativa da diferença entre médias de tratamentos, expressos em percentagem da média (D) pelo método de Hatheway (1961), cuja fórmula é:

$$
D=\sqrt{2\left(t_{1}+t_{2}\right)^{2} A^{2} / r X_{o}^{b}}
$$

em que: r é o número de repetições; Xo é o tamanho ótimo de parcelas, em números de plantas; A e b são estimativas obtidas nas funções $\mathrm{CV}(\mathrm{x})=\mathrm{A} / \mathrm{X}^{\mathrm{B}}$ e $\mathrm{VU}$ $(\mathrm{x})=\mathrm{V}_{1} / \mathrm{X}^{\mathrm{b}} ; \mathrm{t}_{1}$ é o valor tabelado de $\mathrm{t}$ para testes de significância (bilateral a 5\%); e $\mathrm{t}_{2} \mathrm{o}$ valor tabelado de t correspondente a um erro de $2(1-\mathrm{P})$, sendo $\mathrm{P}=0,80 \mathrm{a}$ probabilidade de se obter resultados significativos. Os valores tabelados da distribuição t-Student foram obtidos com GL graus de liberdade, considerando-se o delineamento blocos ao acaso, ou seja, $\mathrm{GL}=(\mathrm{I}-1)(\mathrm{r}-$ 1). O número de tratamentos (I) foi considerado 6 , 12 e 18 , e o número de repetições (r) em $3,5,8$ e 12 . As análises foram realizadas no programa R (R DEVELOPMENT CORE TEAM, 2011) e na planilha eletrônica Office Excel.

\section{RESULTADOS E DISCUSSÃO}

Pela avaliação de cada uma das três áreas como um ensaio de uniformidade no seu respectivo espaçamento entre plantas $(0,4,0,6$ e $0,8 \mathrm{~m})$ foram obtidos os valores de tamanho ótimo de parcela, conquistados pelos dois métodos (MO e MV), com variação de 2,09 plantas para número de rácemos planta ${ }^{-1}$ (MV) até 13,29 plantas para peso de frutos planta ${ }^{-1}$, em gramas (MV) (Tabela 1). O método MV apresenta resultados com maior variabilidade do que o método $\mathrm{MO}$, conforme a característica avaliada, porém não apresentou nenhuma estimativa de tamanho de parcela inferior a uma unidade básica nos três tamanhos de ensaio utilizados, com 432, 288 e 216 unidades básicas.

Em ensaios menores, Lopes et al. (2005) e Martin (2003), com 12 e 96 unidades básicas por ensaio, respectivamente, relatam que o método MV apresentou estimativas do tamanho de parcela inferior a uma unidade básica, considerando esses resultados inadequados.

Oliveira et al. (2006) ressaltam que o método MV depende da escala da unidade de medida usada para a variável, pois ao observar a expressão algébrica para a estimativa de $\mathrm{X} 0$, quando o valor de $\mathrm{V} 1$ for menor do que a unidade, o valor de X0 também vai ser menor do que a unidade. Portanto, bastaria alterar a unidade de medida da variável para resultar em variâncias (V1) maiores do que a unidade e, consequentemente, X0 maiores do que a unidade.

Considerando o método de máxima curvatura modificada (MO), o coeficiente de determinação das funções $\mathrm{CV}(\mathrm{x})=\mathrm{A} / \mathrm{X}^{\mathrm{B}}$ e $\mathrm{VU}(\mathrm{x})=\mathrm{V}_{1} / \mathrm{X}^{\mathrm{b}}$ foi elevado para todas as características avaliadas (Tabela 2), revelando um bom ajuste das funções e estimativas confiáveis dos parâmetros. Observa-se ainda pouca variação do coeficiente de variação entre as parcelas de uma planta (estimativa de A) e do índice de heterogeneidade (estimativa de b) entre espaçamentos, o que significa variabilidade semelhante entre esses.

Para todas as características estudadas o valor de $\mathrm{b}$ foi alto, variando de 0,84 a 1,31, independe do espaçamento utilizado. Nesses casos, conforme estudos de Lin e Binns (1986), a heterogeneidade do solo é alta e os experimentos devem ser estruturados com parcelas maiores e menor número de repetições. Apesar de todas as características, em todos os espaçamentos entre plantas, apresentarem um índice de heterogeneidade alto, parece existir uma tendência linear positiva entre os espaçamentos utilizados e o valor de b, ou seja, quanto mais próximas as plantas menor a variabilidade do ambiente (índice b). É importante ressaltar que as áreas dos três ensaios em branco $(0,4,0,6$ e $0,8 \mathrm{~m})$ foram localizadas lado a lado, porém o número de plantas utilizadas para estimar o b, em cada ensaio, foi diferente $(0,4 \mathrm{~m}=432$ plantas; $0,6 \mathrm{~m}=288$ plantas; e $0,8 \mathrm{~m}=216$ plantas). 
Tabela 1. Tamanho ótimo de parcela em número de plantas usando-se os métodos de máxima curvatura modificada (MO) e máxima curvatura em função da VU (MV) para três espaçamentos entre plantas $(0,4,0,6$ e 0,8 m) e diferentes características em plantas de mamona. Santa Maria (RS), 2015.

\begin{tabular}{ccc}
\hline Espaçamento $(\mathrm{m})$ & MO & MV \\
\hline $0,4 \mathrm{~m}$ & Número de rácemos planta $^{-1}$ & 2,09 \\
$0,6 \mathrm{~m}$ & 8,93 & 2,43 \\
$0,8 \mathrm{~m}$ & 8,95 & 2,45 \\
& 8,49 & \\
$0,4 \mathrm{~m}$ & Peso de frutos planta ${ }^{-1}(\mathrm{~g})$ & 12,15 \\
$0,6 \mathrm{~m}$ & 8,08 & 13,29 \\
$0,8 \mathrm{~m}$ & 8,38 & 11,85 \\
& 7,66 & \\
$0,4 \mathrm{~m}$ & Comprimento médio dos rácemos $(\mathrm{cm})$ & 3,24 \\
$0,6 \mathrm{~m}$ & 7,89 & 3,17 \\
$0,8 \mathrm{~m}$ & 7,48 & 3,06 \\
& 7,52 & 9,80 \\
$0,4 \mathrm{~m}$ & Número total de frutos planta & \\
$0,6 \mathrm{~m}$ & 7,54 & 10,43 \\
$0,8 \mathrm{~m}$ & 8,88 & 9,27 \\
\hline Média geral & 7,91 & 6,94 \\
\hline
\end{tabular}

Tabela 2. Estimativas da média da unidade básica $\left(M_{1}\right)$ dos parâmetros das relações $C V(x)=A / X^{B}$ e VU $(\mathrm{x})=\mathrm{V}_{1} / \mathrm{X}^{\mathrm{b}}$, coeficiente de determinação $\left(\mathrm{R}^{2}\right)$ e do tamanho ótimo de parcela pelo método de máxima curvatura modificada (Xo) para três espaçamentos entre plantas $(0,4,0,6$ e $0,8 \mathrm{~m})$ e diferentes características em plantas de mamona. Santa Maria (RS), 2015.

\begin{tabular}{|c|c|c|c|c|c|c|c|}
\hline Espaçamento(m) & $\mathrm{M}_{1}$ & A & $\mathrm{B}$ & $\mathrm{V}_{1}$ & $b$ & $\mathrm{R}^{2}$ & Xo \\
\hline & \multicolumn{7}{|c|}{ Número de rácemos planta ${ }^{-1}$} \\
\hline 0,4 & 7,11 & 61,17 & 0,42 & 18,93 & 0,84 & 0,94 & 8,93 \\
\hline 0,6 & 9,35 & 61,38 & 0,42 & 32,94 & 0,84 & 0,94 & 8,95 \\
\hline 0,8 & 10,94 & 55,18 & 0,51 & 36,46 & 1,03 & 0,84 & 8,49 \\
\hline Média & 9,14 & 59,24 & 0,45 & 29,44 & 0,90 & 0,91 & 8,79 \\
\hline \multirow[t]{2}{*}{ Conjunto } & - & 56,49 & 0,43 & 32,85 & 0,97 & 0,82 & 8,47 \\
\hline & \multicolumn{7}{|c|}{ Peso de frutos planta ${ }^{-1}(\mathrm{~g})$} \\
\hline 0,4 & 227,91 & 52,47 & 0,44 & 14311,2 & 0,88 & 0,85 & 8,08 \\
\hline 0,6 & 321,48 & 54,25 & 0,50 & 30432,2 & 0,99 & 0,94 & 8,38 \\
\hline 0,8 & 433,45 & 47,05 & 0,60 & 41581,0 & 1,20 & 0,94 & 7,66 \\
\hline Média & 327,61 & 51,26 & 0,51 & 28774,8 & 1,03 & 0,91 & 8,04 \\
\hline \multirow[t]{2}{*}{ Conjunto } & - & 46,61 & 0,47 & 30463,9 & 1,09 & 0,86 & 7,51 \\
\hline & \multicolumn{7}{|c|}{ Comprimento médio dos rácemos $(\mathrm{cm})$} \\
\hline 0,4 & 19,67 & 50,56 & 0,44 & 98,84 & 0,89 & 0,98 & 7,89 \\
\hline 0,6 & 21,21 & 46,17 & 0,47 & 95,84 & 0,95 & 0,97 & 7,48 \\
\hline 0,8 & 22,92 & 45,81 & 0,61 & 110,34 & 1,22 & 0,97 & 7,52 \\
\hline Média & 21,26 & 47,51 & 0,51 & 101,67 & 1,02 & 0,97 & 7,63 \\
\hline \multirow[t]{2}{*}{ Conjunto } & - & 42,80 & 0,46 & 89,15 & 0,95 & 0,80 & 7,07 \\
\hline & \multicolumn{7}{|c|}{ Número total de frutos planta $^{-1}$} \\
\hline 0,4 & 155,07 & 48,11 & 0,42 & 5547,5 & 0,84 & 0,84 & 7,54 \\
\hline 0,6 & 214,25 & 59,12 & 0,54 & 16046,1 & 1,09 & 0,96 & 8,88 \\
\hline 0,8 & 285,04 & 50,23 & 0,66 & 20494,2 & 1,31 & 0,96 & 7,91 \\
\hline Média & 218,12 & 52,49 & 0,54 & 14029,3 & 1,08 & 0,92 & 8,11 \\
\hline Conjunto & - & 47,47 & 0,49 & 13724,7 & 1,12 & 0,87 & 7,65 \\
\hline
\end{tabular}

Revista Caatinga, Mossoró, v. 28, n. 4, p. 253 - 258, out. - dez., 2015 
Considerando a função $\mathrm{CV}(\mathrm{x})=\mathrm{A} / \mathrm{X}^{\mathrm{B}}$ para todas as características e nos três espaçamentos entre plantas, houve pouca variação das estimativas do parâmetro A (variou de 46,17 a $61,38 \%$ ) e B (variou de 0,42 a 0,66$)$, com as quais se estima o tamanho ótimo de parcela. Espera-se, portanto, que o tamanho ótimo de parcela (Xo) também tenha pouca variação em relação aos diferentes espaçamentos entre plantas para as características avaliadas.

Considerando que a média do tamanho ótimo de parcela da análise conjunta de cada ensaio em branco (todos os espaçamentos), pelo método MO, de todas as características (Tabela 2) foi igual a 7,68 unidades básicas (plantas), pode-se inferir que a utilização de oito plantas por parcela é adequada. Devese lembrar de que a área do tamanho ótimo de parcela em cada espaçamento depende da área ocupada pela unidade básica. Assim, a área da parcela com oito plantas é de $3,84,5,76$ e 7,68 $\mathrm{m}^{2}$ para os espaçamentos entre plantas de $0,4,0,6$ e $0,8 \mathrm{~m}$, respectivamente.

A utilização de maior quantidade de plantas na linha (plantas mais próximas, menor valor de b) faz com que sejam necessárias parcelas de menor tamanho. Esse resultado corrobora com as conclusões obtidas por Lopes et al. (2005) para a cultura do sorgo granífero. Juntamente com a menor variabilidade obtida no menor espaçamento entre fileiras, Petinari et al. (2012) encontraram os melhores resultados econômicos quando utilizou espaçamento entre fileiras mais estreitos $(0,45 \mathrm{~m})$, bem como França et al. (2013) quando obtiveram na maior população em estudo (22.000 plantas $\mathrm{ha}^{-1}$ ) os maiores rendimentos. Portanto, no planejamento de experimentos deve-se priorizar, quando possível, a utilização de espaçamentos menores entre plantas, o que torna a área experimental menor, facilitando o trabalho e reduzin- do os custos, sendo também uma alternativa para experimentos conduzidos em áreas restritas.

A estimativa de oito plantas para o tamanho ótimo de parcela, nos diferentes espaçamentos utilizados, permite observar, neste caso, que o mais importante é a variabilidade inerente as plantas e não a área experimental, pois áreas de diferentes tamanhos $\left(3,84 ; 5,76\right.$ e $\left.7,68 \mathrm{~m}^{2}\right)$ contemplam a mesma variabilidade, desde que o número de plantas seja o mesmo.

Considerando experimentos em que se estudem espaçamentos entre plantas, e logicamente com um único tamanho de parcela, possivelmente existirá maior representatividade e/ou precisão nos menores espaçamentos entre plantas (maior número de plantas por parcela) do que nos maiores espaçamentos, o que pode levar a heterogeneidade de variâncias dos erros (LOPES et al., 2005) com efeitos ainda mais evidentes se a parcela experimental for dimensionada para o tamanho ótimo de parcela na condição de menor espaçamento entre plantas.

Com o valor de $A$ da função $\mathrm{CV}(\mathrm{x})=\mathrm{A} / \mathrm{X}^{\mathrm{B}}$ próximo a $50 \%$ e com uma estimativa de $\mathrm{b}$ da função $\mathrm{VU}(\mathrm{x})=\mathrm{V} 1 / \mathrm{X}^{\mathrm{b}}$ próximo a unidade e o tamanho ótimo da parcela igual a oito plantas (tamanho ótimo estimado) foram observadas variações da precisão de experimentos nessa área. Observa-se uma pequena variação da precisão relacionada ao número de tratamentos e grandes variações quanto ao número de repetições para os intervalos estudados (Tabela 3 ). Considerando um coeficiente de variação em torno de $50 \%$ e um índice de heterogeneidade alto $(>0,7)$ (Tabela 3) é possível detectar diferenças entre médias de tratamentos que variam de 19 a $58 \%$ para experimentos planejados com números de repetições entre três e doze e números de tratamentos entre seis e dezoito para características do rácemo de mamoneira.

Tabela 3. Diferença entre médias de tratamentos em percentagem da média (D) para experimentos com variação no número de tratamentos (I) e no número de repetições (r) e respectivos coeficiente de variação $(\mathrm{CV})$ e índice de heterogeneidade (b) para diferentes características de plantas de mamona usando parcelas de oito plantas. Santa Maria (RS), 2015.

\begin{tabular}{|c|c|c|c|c|}
\hline \multirow[b]{2}{*}{$\begin{array}{l}\text { Número de } \\
\text { tratamentos }\end{array}$} & \multicolumn{4}{|c|}{ Número de repetições $(r)$} \\
\hline & 3 & 5 & 8 & 12 \\
\hline & \multicolumn{4}{|c|}{ Número de rácemos planta ${ }^{-1}(\mathrm{CV}=59,24 ; \mathrm{b}=0,9021)$} \\
\hline 6 & 58,8 & 43,2 & 33,4 & 27,0 \\
\hline 12 & 55,5 & 42,0 & 32,9 & 26,7 \\
\hline \multirow[t]{2}{*}{18} & 54,6 & 41,7 & 32,8 & 26,7 \\
\hline & \multicolumn{4}{|c|}{ Peso de frutos planta ${ }^{-1}(\mathrm{CV}=51,26 ; \mathrm{b}=1,0256)$} \\
\hline 6 & 44,8 & 32,9 & 25,4 & 20,5 \\
\hline 12 & 42,2 & 32,0 & 25,0 & 20,3 \\
\hline \multirow[t]{2}{*}{18} & 41,6 & 31,7 & 24,9 & 20,3 \\
\hline & \multicolumn{4}{|c|}{ Comprimento médio dos rácemos $(\mathrm{CV}=47,51 ; \mathrm{b}=1,0184)$} \\
\hline 6 & 41,8 & 30,7 & 23,7 & 19,2 \\
\hline 12 & 39,5 & 29,9 & 23,4 & 19,0 \\
\hline \multirow[t]{2}{*}{18} & 38,8 & 29,6 & 23,3 & 18,9 \\
\hline & \multicolumn{4}{|c|}{ Número total de frutos planta ${ }^{-1}(\mathrm{CV}=52,49 ; \mathrm{b}=1,0787)$} \\
\hline 6 & 43,4 & 31,9 & 24,6 & 19,9 \\
\hline 12 & 40,9 & 31,0 & 24,3 & 19,7 \\
\hline 18 & 40,3 & 30,7 & 24,2 & 19,7 \\
\hline
\end{tabular}

Revista Caatinga, Mossoró, v. 28, n. 4, p. 253 - 258, out. - dez., 2015 


\section{CONCLUSÕES}

O tamanho ótimo de parcela é de oito plantas de mamona, dispostas em uma ou mais linhas, para as características de produção, com a área da parcela de $3,84,5,76$ e $7,68 \mathrm{~m}^{2}$ para os espaçamentos entre plantas de $0,4,0,6$ e $0,8 \mathrm{~m}$, respectivamente.

Considerando um coeficiente de variação em torno de $50 \%$ e um índice de heterogeneidade maior do que 0,7 é possível detectar diferenças entre médias de tratamentos menores do que $27 \%$ para as características de produção em experimentos com 12 repetições.

\section{REFERÊNCIAS}

ALVES, S. M. de F.; SERAPHIN, J. C. Coeficiente de heterogeneidade do solo e tamanho de parcela. Pesquisa Agropecuária Brasileira, Brasília, v. 39, n. 2, p. 105-111, 2004.

CARGNELUTTI FILHO, A. et al. Tamanho de amostra de caracteres em híbridos de mamoneira. Ciência Rural, Santa Maria, v. 40, n. 2, p. 250-257, 2010.

CARVAlHO, E. V. et al. Densidade de plantio em duas cultivares de mamona no sul do Tocantins. Ciência Agronômica, Fortaleza, v. 41, n. 3, p. 387392, 2010.

COMPANHIA NACIONAL DE ABASTECIMENTO (Conab). Safras: série histórica (mamona). 2014. Disponível em: <http://www.conab.gov.br>. Acesso em: 10 jan. 2015.

COMISSÃO ESPECIAL DA BIOENERGIA. Mamona petróleo verde: uma alternativa de verdade. 3 ed. Porto alegre: Assembléia Legislativa do Estado do Rio Grande do Sul, 2006. 53 p. (Documentos, 1).

FRANÇA, R. G. et al. Comportamento de cultivares de mamona (Ricinus communis) sob diferentes densidades populacionais, no estado do Tocantins. Revista de Ciências Agrárias de Portugal, Lisboa, v. 36, n. 3 , p. $317-323,2013$.

GOMEZ, K. A.; GOMEZ, A. A. Statistical procedures for agricultural research, 2. ed. New York: John Wiley \& Sons, 1984. 680 p.

HATHEWAY, W.H. Convenient plot size. Agronomy Journal, Madison, v. 53, n. 4, p. 279-280. 1961.

HOSHMAND, A. R. Design of experiments for agriculture and the natural science. 2. ed. Boca Raton: Chapman \& Hall/CRC, 2006. 456 p.
LIN, C. S.; BINNS, M. R. Relative efficiency of two randomized block designs having different plot size; numbers of replications; of plots per block. Agronomy Journal, Madison, v. 78, n. 3, p. 531-534, 1986.

LOPES, S. J. et al. Tamanho de parcela para produtividade de grãos de sorgo granífero em diferentes densidades de plantas. Pesquisa Agropecuária Brasileira, Brasília, v. 40, n. 6, p. 525-530, 2005.

MARTIN, T. N. Contribuição das bases genéticas de milho para o plano experimental. 2003. $86 \mathrm{f}$. Dissertação (Mestrado em Agronomia) - Universidade Federal de Santa Maria, Santa Maria, 2003.

MEIER, V. D., LESSMAN, K. J. Estimation of optimum field plot shape; size for testing yied in Crambe abyssinica hordnt. Crop Science, Madison, v. 11, n. 5, p. 648-650, 1971.

OLIVEIRA, S. J. R. de et al. Índice de heterogeneidade, coeficiente de variação e tamanho ótimo de parcela em batata. Ciência Rural, Santa Maria, v. 36, n. 6, p. 1710-1716, 2006.

PETINARI, R. A. et al. Custos de produção e lucratividade de cultivares de mamona em diferentes arranjos de plantas. Pesquisa Agropecuária Tropical, Goiania, v. 42, n. 2, p. 143-149, 2012.

R DEVELOPMENT CORE TEAM. R: a language andenvironment for statistical computing. Vienna 2011. Disponível em: <http://www.R-project.org>. Acesso em: 31 jul. 2012.

SCIVITTARO, W. B.; PILLON, C. N. Calagem e adubação para a cultura da mamona no Sul do Brasil. Pelotas: Embrapa Clima Temperado, 2006. 8 p. (Comunicado Técnico, 150).

SMITH, H. F. An empirical law describing heterogeneity in the yield of agricultural crops. Journal Agricultural Science, Cambridge, v. 28, n. 1, p. 1-23, 1938.

STEEL, R. G. D. et al. Principles and procedures of statistics. 3 ed. New York: McGraw Hill, 1997. $666 \mathrm{p}$.

STORCK, L. et al. Experimentação vegetal. 3 ed. Santa Maria: UFSM, 2011. 198 p.

THOMAS, E. J. Relationship between plot size; plot variance. Agricultural Research Journal Kerala, Kerala, v. 12, n. 2, p. 178-189, 1974. 